\title{
A HYBRID AGENT MODEL OF BEHAVIORAL TESTING
}

\author{
Anna Sugak ${ }^{1)}$, Oleksandr Martynyuk ${ }^{2)}$, Oleksandr Drozd ${ }^{3)}$ \\ ${ }^{1)}$ Department of Computerized Control Systems, Odessa National Polytechnic University, \\ Shevchenko Avenue, 1, Odessa, 65044, Ukraine, e-mail: sygak.anna@mail.ru \\ ${ }^{2)}$ Department of Computer Intellectual Systems and Networks, Odessa National Polytechnic University, \\ Shevchenko Avenue, 1, Odessa, 65044, Ukraine, e-mail: anmartynyuk@ukr.net, drozd@ukr.net
}

\begin{abstract}
Operation testing and diagnostic tests, applied for distributed information systems, inherit and employ the properties of distribution, autonomy, goal formation and cooperation, natural for the multi-agent systems. This paper presents the behavioral diagnostics agent model, based on the evolutionary organization of component tests in the automata network environment. The model can be used to construct a multi-agent diagnostics system. A hybrid agent model provides a combination of reactive operation testing and deliberative diagnostic tests, based on the deterministic and evolutionary methods of synthesis of behavioral tests. An agent model consists of the component models of allocation environment, functioning goals and strategies, operations of observation, enforcement strategy and adaptation, initial component models, goals and strategies for ensuring the autonomy. Agent intelligence is based on a locally-exhaustive deterministic and pseudorandom targeted evolutionary synthesis of behavioral tests, providing and accumulating the results. Cooperation of the agents involves their deterministic and evolutionary interactions under the conditions of test feasibility and portability. Copyright (C) Research Institute for Intelligent Computer Systems, 2015. All rights reserved.
\end{abstract}

Keywords: distributed information system, behavioral test, the evolutionary system, agent, multi-agent diagnostics system.

\section{INTRODUCTION}

Rapidly developing modern distributed information systems (DIS) are characterized by $[1,2]$ :

- the emergence of service-oriented architectures, cluster and GRID-systems, cloud and multi-agent technology, used under the conditions of partial definiteness and non-determination;

- the development of mathematical software for the purpose of creation of decomposed, functional, fuzzy, intelligent and competitive models;

- the integration of technologies, architectures and models with the use of common tools and technological means of design and application.

The effectiveness of the DIS depends on the amount of information, quality and reliable performance, efficiency and reliability of the results. The most important way to increase the reliability of the DIS is the technical diagnosing, which is the cornerstone of construction and introductions of the automated technical diagnosis systems (ATDS) $[3,4]$. ATDSs generally include complementary means of operation and test control of DIS efficiency.

At the same time, the DIS improvement promotes the development of the ATDS and the tools for their constructing. As a result, the complexity of ATDS development becomes comparable with the complexity of the DIS. Furthermore, in some cases, it surpasses the last one within the systems of critical application.

These DIS features are inherited by the ATDS models. Their importance is increased according to:

- scalability, distribution, multi-level and multiplatform nature, involving consideration of a great majority of special local features and characteristics;

- complexity, dynamism and incomplete definition, required for the elaboration of behavioral highlevel specifications and formal models of the entire DIS, its subsystems and the component compositions, and non-trivial interfaces of these components, their compositions and subsystems;

- autonomy, cooperation, goal formation, additional mobility, allowing to use the efficient technology of neuron networks and multi-agent systems (MAS) [5-7].

In addition, strong coherence of the DIS and ATDS life cycles, from the design stage is feasible. In particular, system, structural and functional specifications, distribution and multilevel nature of ATDS and DIS allow to use the developed behavioral test software of ATDS to verify the DIS 
projects and ultimately, to use it for the DIS implementation testing $[8,9]$.

Thus, a conclusion can be drawn about the relevance and nontriviality of the solution of the problems of ASTD creation for the complex DIS.

\section{ANALYSIS OF EXISTING SOLUTIONS}

Test synthesis, usually characterized by $N P$-complexity, requirements for the check of high completeness at allowable computing costs $[10,11,12]$, is of great importance for the verification and testing. Development of the models and methods for the automata theory and experiments, determining the general methodology of the behavioral test analysis, removes the upper bounds of applicability of the test automata models $[13,14]$. Complexity reduction, first of all, the reduction of the time of behavioral tests synthesis in a class of errors of mapping with the substantially saved values of the check completeness, is reached by:

- the distributed network $[15,16,17]$ and throughhierarchical [18] methods, reducing polynomially the dimension of the test synthesis and its duration due to the decomposition;

- the evolutionary-genetic methods [19-21], which have the upper bounds of complexity of the determined methods and give polynomially smaller experimental values of complexity of the tests synthesis for the majority of cases [22].

Nevertheless, the synthesis of behavioral tests of the acceptable check completeness is possible for DIS with an average degree of complexity; it refers to the test of $N P$-complex class despite this reduction. This promotes, in particular, the feasibility of further development of the systems of evolutionary-genetic network methods with the combined effect of complexity reduction.

Being a part of real time and critical application systems, a considerable part of modern DIS increases the requirements for completeness, accuracy and relevance of the operation check of efficiency and necessary test resources. This promotes to apply ASTD, including means of operation and test control $[8,11]$, even at the structural and functional system level. At this level the ATDS operation is formally and intuitively based on a system of behavioral testing methods [23].

The possibility of passive mode operation control and active mode test control is a characteristic feature of behavioral tests [13]. Passive mode deals with the current accumulation of presentable passive recognizing experiments, as a background process of the DIS components normal operation functioning. The interruption of the operation functioning occurs during the active mode of test control, thus, the control test verification experiments are conducted. Both cases are accompanied, if necessary, by the previous internal or external test synthesis.

However, insufficiently investigated formalization of walkthrough comprehensive operation and test behavioral control and ambiguous definition of the verifiable errors class doesn't allow ATDS to control effectively over the received values of the system check completeness, accuracy and relevance and functional verification of DIS. It promotes further development of the systems of the network determined and evolutionary-genetic methods, the solution of the problems of their allocation in the DIS environment, initialization and cooperative performance.

Currently, there are increasingly effective ATDSs of distributed systems, using models and methods of multi-agent technology [24-26]. Nevertheless, there are issues of multi-agent analysis that have to be developed, such as network behavior analysis, cooperation and intelligence of their agents, especially in the case of:

- incomplete determination of testable properties (errors), reducing the check completeness

- network control, limited by separate rules, and monitoring of the diagnosis, that narrows the space of behavioral tests search;

- fixed combination of deterministic and evolutionary methods, complicating the dynamic adjustment to situationally created cooperation;

- a lack of operation and test control, eliminating the background behavioral testing in operation mode and requiring for check completeness in the operation test pauses.

Thus, we can draw a conclusion about the usability of research of the system and functional control increasing in the error class of the DIS mapping and the development of the agent model of the DIS comprehensive operation and test behavioral control, based on the deterministic and evolutionarygenetic network methods, implemented in the ATDS.

\section{GOAL AND TASKS}

The DIS behavioral model as the network of automata (NA) is researched as the MAS environment model and is characterized by structure, alphabets and compliances of components, data and knowledge structures, which can be altered in the DIS life cycle.

The MAS operation and test control in DIS is represented by agents Ag cooperation, placed in the DIS environment. The MAS atomic element $a g_{i} \in A g$ presents the first-level MAS model.

The purpose of constructing of a certain agent $a g_{i}$ model is formalization of presentation of the behavioral agent-based operation and test control. 
This control is performed on the base of the automata model $a_{i} \in A$ for the components of DIS within the contexts:

- autonomous;

- the goal forming or intellectual;

- cooperative.

The context of mobility is not being considered in this paper.

The majority of the tests of the DIS behavioral control performed by the agent $a g_{i}$ of the model of automata $a_{i}$, includes:

- construction of identifiers of $T i_{i}$ resistance states;

- construction of test $T p_{i}$ and binders $L p_{i}$ primitives;

- passive component recognition of text fragments $T f_{i}$ up to the behavioral tests of DIS component for its operation control;

- the active formation of component test fragments $T f_{i}$ up to the behavioral tests of the DIS component for its test control;

- forming of the component experiments - test experiments of deterministic $\operatorname{texp}_{D i} \in T \operatorname{Exp}_{D}$, evolution $\quad \exp _{E i} \in T E x p_{E}, \quad$ combined $\operatorname{texp}_{D E i} \in T \operatorname{Exp}_{D E}$ behavioral testing for the agent $a g_{i}$;

- construction of component minimized, input implemented $a R_{i}$ and output transported $a T r_{i}$ semi-automata, nodal implemented $a R_{T}^{X-1}$ (ai) and nodal transported $\operatorname{aTr}_{T(a i)}^{Y}$ semi-automata, based on the relevant input $T^{-1}$ (ai) and output $T$ (ai) nodal subnets of the DIS components topology;

- multi-agent decomposition task of behavioral testing of the DIS components, in particular, the synthesis of the component behavioral tests on a set of the agent testing Task $_{T}$, implementing Task $_{R}$, transporting Task $k_{T r}$ and dispatching Task;

- definition of the system of sets Rel $_{\text {Set }}$, -component -object $\operatorname{Rel}_{O b j C o m}$, structural $\operatorname{Rel}_{\text {struct }}$, functional $\operatorname{Rel}_{\text {Func }}$, temporal $\operatorname{Rel}_{\text {Time }}$ relations, in particular, precedence relationships Rel $_{\text {Pref }}$ for the agentbased tasks Task Task $_{R}$, Task $_{T r}$ and Task

- construction of structure $G_{\text {Task }}\left(\left\{\right.\right.$ Task $_{T}$, Task $k_{R}$, Task $\left.\left._{T r}, T_{\text {Task }}\right\}, \Delta_{\text {Task }}\right)$ of the agent tasks Task ${ }_{T}$, Task $_{R}$, Task $_{T r}$ and Task $k_{S}$, based on a system of sets $\operatorname{Rel}_{\text {Set }}$, component -object $\operatorname{Rel}_{\text {objCom }}$, structural $\mathrm{Rel}_{\text {Struct, }}$, functional $\mathrm{Rel}_{\text {Func }}$, temporal $\mathrm{Rel}_{\text {Time }}$ relations;

- resources definition $\left\{R_{\text {TaskT }}, R_{\text {TaskR }}, R_{\text {TaskTr }}, R_{\text {Task } S\}}\right\}$, required for the structure $G_{\text {Task }}\left(\left\{\right.\right.$ Task $_{T}$, Task $k_{R}$, Task $\left._{T r}, T_{\text {Task }}\right\}, \Delta_{\text {Task }}$ ) of the agent tasks Task ${ }_{T}$, Task $_{R}$, Task $_{T r}$ and Task ${ }_{S}$ and available for the agents;

- forming of the component test experiments of deterministic $\quad \exp _{D i} \in T \operatorname{Exp}_{D}$, evolutionary $\operatorname{texp}_{E i} \in T E \operatorname{Exp}_{E}$, combined $\operatorname{texp}_{D E i} \in T \operatorname{Exp}_{D E}$ behavioral testing for the agents $a g_{i} \in A g$ MAC;
- task scheduling Task $_{T}$, Task $_{R}$, Task $k_{T r}$ and Task, scheduling dispatching of the agent resources $\left\{R_{\text {TaskT }}, R_{\text {TaskR }}, R_{\text {TaskTr }}, R_{\text {TaskS }}\right\}$ on the basis of the technical solutions - input buffers of the tasks, dynamic priorities of the tasks, mechanisms of critical sections of resources, quantization of access, transaction for behavioral testing tasks, output buffers of solutions in accordance with the component test experiments $T E x p=T \operatorname{Exp}_{D} \cup T \operatorname{Exp}_{E} \cup T E x p_{D E}$.

\section{AGENT MODEL CONSTRUCTION}

Hybrid type agent $a g_{i}$, comprising two complementary components - reactive deterministic $a g_{R i}$ and deliberative evolutionary $a g_{D i}$, in general case is a system-five:

$$
a g_{i}=\left(a g_{R i}, a g_{D i}, X_{i}, Y_{i}, \Delta_{i}\right) \text {, }
$$

where $a g_{R i}, a g_{D i}$ correspondingly reactive and deliberative components - interacting subsystems, parallel (in environment) and/or sequential (in time), functioning within the corresponding component of DIS, sharing the inputs $X_{i}$ (conditions-events) and outputs $Y_{i}$ (actions) of the agent $a g_{i} ; \Delta_{i}$ - general function of the components interaction.

The priority of choosing of the reactive $a g_{R i}$ or deliberative $a g_{D i}$ component and its subsequent activization are defined by the solution of a random test $\operatorname{Task}_{T}$ task, according to the type, complexity and condition of the corresponding component automata ai, representing a random component of DIS, the MAS condition and goal formation of the corresponding agent $a_{i}$.

The generality of the test data and knowledge models for the reactive $a g_{R i}$ or deliberative $a g_{D i}$ components of the hybrid agent $a g_{i}$ reduces the difference between them to the peculiarities of their methods of behavioral testing:

- additional - in a deterministic or evolutionary construction and recognition of identifiers $T i_{i}$, tests $T p_{i}$ and links $L p_{i}$ primitives, in a deterministic or evolutionary construction of the inverse input implemented $T^{-1}\left(a_{i}\right)$ and direct output transported $T\left(a_{i}\right)$ nodal subnets, implemented $a R_{T}^{X-1}$ (ai) and transported $a \operatorname{Tr}_{T(a i)}^{Y}$ nodal sub-automata;

- basic - in a deterministic or evolutionary construction and recognition of the test fragments $T f_{i}$ and in a deterministic or evolutionary construction of the test experiments $T \operatorname{Exp}_{i}$.

Extended agent model $a g_{i}$ (with reactive $a g_{R i}$ and/or deliberative $a g_{D i}$ components) having information about its own input $T^{-1}\left(a_{i}\right)$ and output $T\left(a_{i}\right)$ nodal subnets NA, and about the implemented $a R_{T}^{X}{ }_{T}$ (ai) and transportable $a \operatorname{Tr}_{T(a i)}^{Y}$ nodal subnets of sub-automata, is represented by: 


$$
a g_{i}=\left(M_{i}, Q_{i}, S t_{i},\left\{o_{i}, \sigma_{i}, \alpha_{i}\right\},\left\{m_{0 i}, q_{0 i}, s t_{0 i}\right\}\right),
$$

where $M_{i}$ - a set of the agent-based models of the placement component of DIS; $Q_{i}-$ a set of the agents goals, for certain $q_{i} \in Q_{i}$ defined as $q_{i}: M_{i} \times M_{i} \rightarrow D_{i}$ :

$$
\begin{gathered}
q_{i}\left(m_{i}, m_{i}{ }^{\prime}\right)=\left(k_{\varphi v i}\left(\varphi v_{i}\left(m_{i}{ }^{\prime}\right)-\varphi v_{i}\left(m_{i}\right)\right), k_{\lambda \varepsilon i}\left(\lambda \varepsilon_{i}\left(m_{i}{ }^{\prime}\right)-\right.\right. \\
\left.-\lambda \varepsilon_{i}\left(m_{i}\right)\right), k_{\theta v i}\left(\theta v_{i}\left(m_{i}{ }^{\prime}\right)-\left(\theta v_{i}\left(m_{i}\right)\right)\right),
\end{gathered}
$$

where, $\varphi v_{i}, \lambda \varepsilon_{i}, \theta v_{i}, \rho \varepsilon_{i}, \tau \rho_{i}-$ detection functions of the check completeness, length, multiplicity, feasibility and portability of a test, $m_{i}$ and $m_{i}{ }^{\prime}-$ correspondingly the initial and the following estimated agent-based models of the placement component; $S t_{i}-$ a set of strategies of agent functioning, for a certain $s t_{i}$ defined as $s t_{i}: M_{i} \rightarrow M_{i}$, $m_{i}{ }^{\prime}=s t_{i}\left(m_{i}\right) ;\left\{\begin{array}{lll}o_{i}, & \sigma_{i}, & \alpha_{i}\end{array}\right\}$ - the signature of the agent operations, correspondingly:

a) observation $\quad o_{i}: M_{i} \times V_{i} \rightarrow M_{i}, \quad m_{i}=o_{i}\left(M_{i}, V_{i}\right)$, determining of the operation agent model and forming, if it is necessary, of the identifiers $T i_{i}$ and the test primitives $T p_{i}$, where $V_{i}=\left(e n_{i}, a g_{i}\right.$, $\left.\mathrm{Conn}_{i}\right)$ - agent environment $a g_{i}$, for which:

1) $e n_{i}=\left(a_{i}, P l_{i}\right)$ - agent-world environment for the agent $a g_{i}$

2) $C o n n_{i}$ - relationship between the agent $a g_{i}$ and the environment $e n_{i}$;

3) $P l_{i}=\left\{p l_{\text {ini }}, p l_{\text {outi, }}, p l_{\text {inouti, }} p l_{\text {loopbacki }}\right\}$ - a set of the basic agent placement for $a g_{i}$ in environment $e n_{i}$;

b) implementation of the strategy $\sigma_{i}: S t_{i} \times V_{i} \rightarrow V_{i}$, $V^{\prime}=\sigma_{i}\left(s t_{i}, V_{i}\right)-$ creation of modified environment $V_{i}^{\prime}$ and agent $a g_{i}^{\prime}$ in its structure, definition of link primitives $L p_{i}$, construction of the test fragments $T f_{i}$ and definition of the completeness $\varphi v_{i}$, length $\lambda \varepsilon_{i}$, multiplicity $\theta v_{i}$, feasibility $\rho \varepsilon_{i}$ and portability $\tau \rho_{i}$ of a set of the received test fragments for the operation agent model $m_{i}$;

c) adaptation $\quad \alpha_{i}=\left(\alpha_{m i}, \quad \alpha_{s i}\right), \quad$ where $\alpha_{m i}: M_{i} \times M_{i} \rightarrow M_{i}, \quad M_{i}{ }^{\prime}=\alpha_{m i}\left(M_{i}, \quad m_{i}, \quad m_{i}{ }^{\prime}\right) \quad$ and $\alpha_{s i}: S t_{i} \times M_{i} \rightarrow S t_{i}, S t_{i}{ }^{\prime}=\alpha_{m i}\left(S t_{i}, m_{i}, m_{i}{ }^{\prime}\right)$ - fixation of updating of the sets of strategies $S t_{i}$ and agentbased models of the component placement $M_{i}$, composed of:

1) initial identifiers $T i_{i}$ and test primitives $T p_{i}$;

2) test fragments $T f_{i}$;

3) input implemented $a R_{i}^{X}$ and output transported $\operatorname{aTr}_{i}^{Y}$ component semi-automata;

4) input implementing $T^{-1}\left(a_{i}\right)$ and output transporting $T\left(a_{i}\right)$ nodal subnets;

5) input implemented $a R_{T}^{X-1}(a i)$ and output transported $a \operatorname{Tr}_{T(a i)}^{Y}$ nodal semi-automata;

- $\left\{m_{0 i}, q_{0 i}, s t_{0 i}\right\}$ - the initial model, goal and strategy of the agent.

A certain placement agent model $m_{i} \in M_{i}$ of the above mentioned set of models $M_{i}$ is defined as:

$$
\begin{gathered}
m_{i}=\left(a_{i}, T_{i}, \operatorname{Tp}_{i}, \operatorname{Tf}_{i}, \operatorname{aR}_{i}^{X} \operatorname{aTr}_{i}^{Y}, T^{-1}\left(a_{i}\right), T\left(a_{i}\right),\right. \\
\left.a R_{T}^{X}{ }_{T}^{-1}(a i), \operatorname{aTr}_{T(a i)}^{Y}\right),
\end{gathered}
$$

where for a certain DIS component of the automata $a_{i}$ :

- $a_{i}$ - tested automata of the environment component, designated for the agent, which can be a sub-automata of the entire component automata model;

- $T i_{i}$ - initial identifiers of the support states and $T p_{i}-$ test primitives;

- $T f_{i}$ - test fragments;

- $a R^{X}{ }_{i}$ - the component input implemented subautomata and $a \operatorname{Tr}^{Y}{ }_{i}$ - the component output transported sub-automata;

- $T^{1}\left(a_{i}\right)$ - the node input implementing subnet of a network NA and $T\left(a_{i}\right)$ - the node output transporting subnet of a network NA;

- $a R_{T}^{X-1}(a i)$ - the node input implemented semiautomata and $\operatorname{aTr}_{T(a i)}^{Y}$ - the node output transported semi-automata.

The specificity of the functioning of the reactive component $a g_{R i}$ of an agent $a g_{i}$ - the performance of its operations $\left\{o_{i}, \sigma_{i}, \alpha_{i}\right\}$, is based on the deterministic test methods with the search to depth or/and width. These methods are applied to achieve a local-exhaustive optimization. They are based on the automata experiments $[5,9,10]$ and characterized by $N P$-complexity of testing, in particular, by the synthesis of behavioral tests.

This fact implies the restriction of the analysis (a subset of the components of DIS) by the medium complexity (up to 1000 states) to obtain the solution of the test tasks, as a rule, of the required high completeness $\varphi v_{i}$, acceptable length $\lambda \varepsilon_{i}$ and multiplicity $\theta v_{i}$, with time $\tau v_{i}$ and memory $\mu \varepsilon_{i}$, limited by upper bounds of dedicated computing resources $R_{\text {agRi }}=\left(\tau l_{\text {MaxagRi, }} \mu \varepsilon_{\text {MaxagRi }}\right)$ of reactive component $a g_{R i}$.

The specificity of the functioning of the deliberative component $a g_{D i}$ of the agent $a g_{i}$ - the performance of its operations $\left\{o_{i}, \sigma_{i}, \alpha_{i}\right\}$ is based on the pseudo-random, goal-oriented test search of the evolutionary-genetic approach [27]. Therefore, for automata test experiments, these methods retain the upper exponential analytical evaluations of deterministic methods, but their experimental complexity is significantly less than computed $N P$-complexity.

This leads to a solution of tasks of the acceptable composition with resources of time and memory, less than of the middle range, which is limited by lower and upper boundaries of resources $R_{\text {agDi }}=\left(\begin{array}{ll}\tau \iota_{\text {Maxag } D i} & \mu \varepsilon_{\text {MaxagD } i}\end{array}\right) \quad$ of deliberative component, in the space of analysis (a subset of the components of DIS) of objects which is above the average degree of complexity (more than 1000 automata states). 
Intelligence of the agent $a g_{i}$ (for its deliberative component $\left.a g_{D i}\right)$ is based on the evolutionary systems of behavioral tests synthesis [27]:

$$
T e_{i}=\left(T f_{i}, T p_{i}, L p_{t i}, S g_{t i}, T f_{f i}\right),
$$

where $T f_{i}, T p_{i}, L p_{t i}-$ a set of test fragments, the initial set of test primitives $T p_{i}=T f_{0 i} \subseteq T f_{i}$, linking primitives $L p_{t i}, T f_{f i}$ - the final set of test fragments; $S g_{\text {tag } D i}=\left\{\mu_{\text {tagDi } i}, \kappa_{\text {tag } D i}, \phi_{\text {tag } D i}, \varphi_{\text {tag } D i}, \sigma_{\text {tagDi }}\right\}$ - signature of the operations and functions of the test evolution, consisting of the test mutation, crossover, immunity, fitness and selection functions.

Besides the test objects of population $T_{+i}$, the model proposes syntactically and functionally similar to them additional infectious and vaccine objects [27]. Infectious objects $\Omega_{+i}=\Omega l_{i} \cup \Omega p_{i} \cup \Omega f_{i}$, as test objects, may include identifiers $\Omega t_{i}$, primitives $\Omega p_{i}$, fragments $\Omega f_{i}$ and the population, as a whole $\Omega_{+i}$, and form a system of fragments with signature of operations and functions in the evolution $\Omega e_{i}$. Infectious objects $\Omega_{+i}$ are generated within the infectivity evolution, external for the test evolution.

Formation and formal representation of infectious objects $\Omega_{+i}$ is similar to the presentation and formation of the relevant test objects $T_{+i}$. However, it is characterized by its own, though similar, definitions, operations and functions for the infectious objects.

Infectious objects follow $\Omega_{+i} \subseteq W_{i}{ }^{\text {"^, }}, W_{i}{ }^{\text {"’^ }}$ - the behavior of the infectious automata $a_{i}^{\wedge}$, for which discrepancy (or non inclusion) is possible in relation to the tested automata $a_{i}$, that is, it is possible $W_{i}{ }^{\prime \prime} \neq \neq W_{i}$, (or $W_{i},{ }^{\prime} \wedge \mid W_{i}, " \neq \varnothing$ ).

Infectious objects $\Omega_{+i}$ have a form, similar to their analogs - test objects from $T_{+i}$ :

$$
\begin{aligned}
& \omega u_{i}^{S^{\wedge}}=\left(T_{i}, W_{i}{ }^{\prime \wedge}, S_{i}^{\wedge}, \Delta_{i}, \Lambda_{i}^{S^{\wedge}}\right), \\
& \omega p_{i}{ }^{{ }^{\wedge}}=\left(T_{i}, W_{i}{ }^{\prime \wedge}, S_{i}^{\wedge}, \Delta_{i}, \Lambda_{i}^{S^{\wedge}}, \Omega l_{t p i}^{S^{\wedge}}, W_{i}{ }^{,}{ }^{\wedge S E}\right), \\
& \omega f_{i}^{W^{\wedge}}=\left(T_{i}, W_{i}{ }^{\prime \prime}, S_{i}^{\wedge}, \Delta_{i}, \Lambda_{i}^{S^{\wedge}}, \Omega p_{t f i}^{S^{\wedge}}, L p_{t f i}^{S^{\wedge}}, W_{i}{ }^{\prime}, S E\right),
\end{aligned}
$$

where models components are presented with the definition of the test objects, the model of the infectious evolution synthesis for $a_{i}^{\wedge}$ is defined as infectious evolution of the common type with the test evolution:

$$
\Omega e_{i}=\left(\Omega f_{i}, \Omega p_{i}, L p_{\omega i}, \Omega i, S g_{\omega i}\right),
$$

where $\Omega f_{i}, \Omega p_{i}, L p_{\omega i}, \Omega i_{i}$ - are presented above the sets of correspondingly infectious fragments, initial set (in evolution) of infectious primitives $\Omega p_{i}=\Omega f_{0 i \subseteq} \subseteq f_{i}$ and linking primitives, a set of infectious identifiers $\Omega i_{i} \neq W_{i}{ }^{\prime \prime}$ (or $\Omega i_{i} \mid W_{i}{ }^{\prime \prime} \neq \varnothing$ ); $S g_{\omega i}=\left\{\mu_{\omega i}, \kappa_{\omega i}, \varphi_{\omega i}, \sigma_{\omega i}\right\}$ - signature of infective evolution, such as a test signature.
For infectious $\Omega e_{i}$ and test $T e_{i}$ evolutions the interaction of certain objects - identifiers, primitives, fragments - are based on monobasic (inside $\Omega e_{i}$ or $T e_{i}$ ) operations of crossover $\left\{\kappa_{t i}, \kappa_{\omega i}\right\}$ or dibasic $\left(\Omega e_{i} \times T e_{i}\right)$ operations of mutation $\left\{\mu_{t i}, \mu_{\omega i}\right\}$. Operations are preceded by definition of the fitness $\left\{\varphi_{t i}, \varphi_{\omega i}\right\}$ and selection $\left\{\sigma_{t i}, \sigma_{\omega i}\right\}$ functions with deterministic and pseudorandom priority settings $\Pi_{t i}$, $\Sigma_{t i}, \Pi_{\omega i}, \Sigma_{\omega i}$.

The vaccine objects - identifiers $\Psi t_{i} \subseteq W_{i}$ "^, primitives $\Psi p_{i} \subseteq W_{i}{ }^{\prime \prime}$, fragments $\Psi f_{i} \subseteq W_{i}{ }^{\prime \prime} \wedge$ and population $\Psi_{+i} \subseteq W_{i}$ "'^ - are located in the space $W_{i}{ }^{\text {"'^},}$, which is enlarged correspondingly to $a_{i}$, as well as infectious objects, generating them (knowledge and recognition of infections). The vaccine objects, expanding the conventional test objects, accumulate a successful immune experience. We can talk about the inclusion of $\Psi l_{i} \subseteq T i_{i}^{\tilde{i}}, \quad \Psi p_{i} \subseteq T p_{i}^{\tilde{i}}, \quad \Psi f_{i} \subseteq T f_{i}^{\sim}, \quad \Psi e_{i} \subseteq T e_{i}^{\tilde{i}}$ and in general $\Psi_{+i} \subseteq T_{+i} \sim$, where $\Psi_{+i}=\Psi i_{i} \cup \Psi p_{i} \cup \Psi f_{i}$, into the context of the current status of the population $T_{+i} \tilde{}$ of the evolution development $T e_{i}$, because the $\Psi_{+i}$ is a part of test $T_{+i}$, formed by infections.

Therefore, the complex model of the test $C e_{i}$ synthesis for $a_{i}$ is defined as the co-evolution internal complex evolution in the space of test $T e_{i}$ and infectious $\Omega e_{i}$ evolutions:

$$
C e_{\Omega i}=\left(T e_{i}, \Omega e_{i}, \Psi_{\Omega+i},\left\{\mu_{\psi i}, \kappa_{\psi i}\right\}, \Phi_{\Omega i}, T f_{f i}\right),
$$

with the selection of vaccine population $\Psi_{+i}$ in $T e_{i}$, a signature of the internal mutations operations $\mu_{\psi i} \subseteq \mu_{t i} \cup \mu_{\omega i}$ and presumably a crossover $\kappa_{\psi i} \subseteq \kappa_{t i} \cup \kappa_{\omega i}$, immune search function $\Phi_{\Omega i}=\left\{\phi_{\Omega+i}\right.$, $\phi_{T+\sim i(\Omega+\sim i)}, \phi_{\text {Premui }}^{-1}$, and the final set of test fragments (test population) $T f_{f i}$.

Agent cooperation of evolutions for the test $T e_{i}$ or viral-test $C e_{i}$ is possible as external interactions with evolutions of the other agents, placed on the other components of DIS.

In this case, the feature of "bonding" of the fragments in the operations of the new external mutation $\mu_{\psi i}^{\prime}$ and crossover $\kappa_{\psi i}^{\prime}$ can be shown through the identity or compatibility (intersection) of the adjacent projections «pr» of basic behaviors of adjacent components in the composition NA - MAS pairs of agents, such as:

- test output $p r_{2}\left(T f_{1 i}\right)$ for $a_{1 i}$ automata of previous $1 i$-component and the test input $\operatorname{pr}_{1}\left(T f_{2 i}\right)$ for $a_{2 i}$ automata of $2 i$-current component $p r_{2}\left(T f_{l i}\right)=p r_{1}\left(T f_{2 i}\right)$;

- implemented output $p r_{2}\left(R f_{j}\right)$ for the automata $a_{j}$ of the previous $j$-component and test input $p r_{1}\left(T f_{i}\right)$ for automata $a_{i}$ of current $i$-component $p r_{2}\left(R f_{j}\right)=p r_{l}\left(T f_{i}\right)$;

- test output $\operatorname{pr}_{2}\left(T f_{i}\right)$ for automata $a_{i}$ of current $i$-component and transported input $\operatorname{pr}_{l}\left(\operatorname{Trf}_{k}\right)$ for 
automata $a_{k}$ of the next $k$-component $\operatorname{pr}_{2}\left(T f_{i}\right)=p r_{1}\left(\operatorname{Tr} f_{k}\right)$.

In addition, new external functions of fitness $\varphi_{\psi i}$ ' and selection $\sigma_{\psi i}$ ' include not only the examination of the criteria of completeness $\varphi v_{i}$, length $\lambda \varepsilon_{i}$ and multiplicity $\theta v_{i}$ of the test patches and multiplicity $\theta v \varepsilon_{i}$ of population, but also the criteria of feasibility $\rho \varepsilon_{i}$, and portability $\tau \rho_{i}$. In the simplest case, these criteria are of the semaphore format, restricting or blocking the application of implemented and/or transported fragments.

In this case, the co-evolutionary interaction is identical with the co-evolutionary viral-test interaction with the allocation of vaccine population $\Psi_{+i}, \mu_{\psi i}{ }^{\prime} \subseteq \mu_{t 1 i}{ }^{\prime} \cup \mu_{t 2 i}$ ' in $T e_{i}$, signatures of external operations of mutation and presumably a crossover $\kappa_{\psi i}{ }^{\prime} \subseteq \kappa_{t 1 i}{ }^{\prime} \cup \kappa_{t 2 i}$ ', immune search function $\Phi_{T i}=\left\{\phi_{T+\sim i}\right.$,

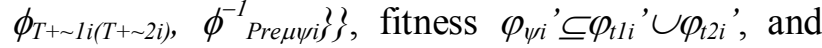
selection $\sigma_{\psi i}{ }^{\prime} \subseteq \sigma_{t 1 i}$ ' $\cup \sigma_{t 2 i}$ ' functions and the fragments $T f_{f i}$ of final population $T_{+f i}$ of test evolution $T e_{i}[28]$ :

$$
C e_{T 12 i}=\left(T e_{1 i}, T e_{2 i}, \Psi_{T+i},\left\{\mu_{\psi i}, \kappa_{\psi i}^{\prime}{ }^{\prime}\right\}, \Phi_{T i}, T f_{f i}\right)
$$

At first, input implementing $R e_{j}$ and transporting Tre $_{k}$ evolutions are formed in co-evolutions $C e_{R j T i}$ and $C e_{T i T r k}$ for $T e_{i}$. This creates input implementing $T^{-1}\left(a_{i}\right)$ and output transporting $T\left(a_{i}\right)$ as two-level nodal subnets, and input implemented $a R_{T}^{X-1}$ (ai) and output transported $a \operatorname{Tr}^{Y}$ (ai) as nodal semi-automata.

After this construction or simultaneously with it, co-evolutions $C e_{R j T i}$ and $C e_{\text {TiTrk }}$ are formed, correspondingly as implemented and transported restrictions of the evolution $T e_{i}$. Then at the same time implemented and transported restrictions of $C e_{T i}$ of evolution $T e_{i}$ appear as the intersection of coevolutions $C e_{R j T i}$ and $C e_{\text {TiTrk }}$ with their possible optimization - common objects determination and their dual use (serial or parallel) as:

- identifiers $T i_{i}$;

- $\quad$ primitives $T p_{i}$ and $L p_{i}$;

- fragments $T f_{i}$;

- two-level subnets $t^{-1}\left(a_{i}\right), t\left(a_{i}\right)$ in subnets $T^{-1}\left(a_{i}\right)$, $T\left(a_{i}\right)$

- two-level sub-automata $a r_{T}^{X-1}(a i), a t r_{T(a i)}^{Y}$ in semiautomata $a R_{T}^{X-1}(a i), a \operatorname{Tr}_{T(a i)}^{Y}$, according to the subnetworks $t^{-1}\left(a_{i}\right), t\left(a_{i}\right)$.

Appropriate signatures of search functions $\Phi_{T i}$, $\Phi_{R j T i}, \Phi_{\text {TiTrk }}$ are identical with the signatures of the immune search generic function $\Phi_{T i}$ of internal coevolution $C e_{T i}$ for external cooperative co-evolutions $C e_{T 12 i}, C e_{R j T i}, C e_{T i T r k}$ and test evolutions $T e_{1 i}, T e_{2 i}$. They provide recognition of input operands of component mutation $\mu_{t i}{ }^{\prime}$ and a crossover $\kappa_{t i}{ }^{\prime}$. It is possible when there is an appropriate experience which can be stored. Therefore, the prepared (stored) results $\left(T i_{i}, T p_{i}, L p_{i}, T f_{i}, T^{-1}\left(a_{i}\right), T\left(a_{i}\right), a R_{T}^{X-1}(a)\right.$, $\left.\operatorname{aTr}_{T(a i)}^{Y}\right)$ can be applied.

Thus, the cooperativeness of the component agent $a g_{i}$, its ability to participate in forming and providing of the internal $C e_{i}$ and external $C e_{T 12 i}$, $C e_{\text {RjTi }}, C e_{\text {TiTrk }}$ cooperation is based on the construction of component minimized input implemented $a R_{T}^{X-1}$ (ai) and output transported $a \operatorname{Tr}_{T(a i)}^{Y}$ nodal semi-automata. They are the key elements of the implemented and transported behavior passing forward and backward in the NA composition through the DIS components.

Therefore, the testing model MAS (of the second level) is a cooperation of $C e_{i}, C e_{T 12 i}, C e_{R j T i}, C e_{\text {TiTrk }}$ of the hybrid agents $A g=\cup_{i \in I} a g_{i}$. In accordance with the problem, which has to be solved, this model deterministically and/or evolutionarily forms and activates the required test structure into a set of reactive $A g_{R}$ and deliberative $A g_{D}$ components of the agents $A g$. These agents are placed in automata of NA - in the DIS components.

The purpose of the external test cooperation of co-evolutions $C e_{T 12 i}, C e_{R j T i}, C e_{T i T r k}$ is tetra-evolution $C e_{\text {RjTiTrk, }}$ as a joint cooperation of agents $C e_{i}, C e_{T 12 i}$, $C e_{\text {RjTi }}, C e_{\text {TiTrk }}$. Tetra-evolution $C e_{\text {RjTiTrk }}$ is the formal representation of the structural and topological, implemented and transported, goal-oriented and intelligent performance of the tasks during the operation and test control of the DIS components.

\section{IMPLEMENTATION AND SIMULATION}

The basic component-object (programming technology) programs in MS Visual.Net environment were offered for the experimental implementation of the agent model $a g_{i}$, with the reactive $a g_{R i}$ and deliberative $a g_{D i}$ components (parts of the agent), which model behavioral testing of the DIS component, performed by the MAS agent. The deterministic and evolutionary-genetic generators and the agent supervisor are selected as the first stage program.

Simulation of deterministic generator is based on a set of component implementations of deterministic models and methods of passive and active synthesis of behavioral tests $\operatorname{Tex}_{i}$, which form a technological structure (system, conditional procedure) of deterministic construction of the test fragments $T f_{i}$ (see Fig. 1). The composition and communication of this structure are defined by the solved deterministic tasks and the relations between them.

The set of basic procedures defines the operation of the deterministic generators during the performance of the test tasks.

The general procedure of the preprocessor test synthesis of behavioral tests Tex $_{i}$ for automata model $a_{i}$ of DIS component includes: 
1) preliminary structural and topological (graph) analysis with the detection in the graph of the component automata $a_{i}$ of inputs, outputs, chains (straight-line paths), trees, hammocks, cycles, the formation of condensation with folded chains and cycles;

2) preliminary definition of identifiers $T i_{i}$ supporting states ( $N P$-complexity), linking $L p_{i}$ and test $T p_{i}$ primitives based on them; preview definition of the input $a^{X}{ }_{i}$ and output $a_{i}^{Y}$ component semiautomata;

3) preliminary definition of the transported (recognizable) component sub-automata $\operatorname{aTr}^{Y}{ }_{i}$ (NP-complexity).

The general procedure of the preprocessor test synthesis of behavioral tests for the model of the DIS automata network includes:

1) preliminary structural and topological (graph) analysis, identifying the inputs, outputs, chains (straight-line paths), trees, hammocks, cycles, the formation of condensation with folded chains and cycles in a graph of the automata network NA;

2) forward and reverse recursive construction of the implementing $T^{-1}\left(a_{i}\right)$ and transporting $T\left(a_{i}\right)$ nodal subnets;

3) forward recursive construction of the implementable nodal semi-automata $a_{X}^{R}{ }^{T-}{ }_{1(a i)}$ based on implementing nodal subnets ${ }^{T-1}(a i)$;

4) reverse recursive construction of the transported nodal semi-automata $\operatorname{aTr}_{T(a i)}^{Y}$ based on transporting nodal subnets $T\left(a_{i}\right)$;

5) narrowing of the identifiers $T i_{i}$ of the supporting states, linking $L p_{i}$ and test $T p_{i}$ primitives receiving of the implemented and transported identifiers $T i_{R j T i T r k}$, linking $L p_{R j T i T r k}$ and test $T p_{R_{B T i} T \text { rk }}$ primitives based on the implemented $a R_{T}^{X-1}(a i)$ and transported $a \operatorname{Tr}_{T(a i)}^{Y}$ of the nodal semi-automata.

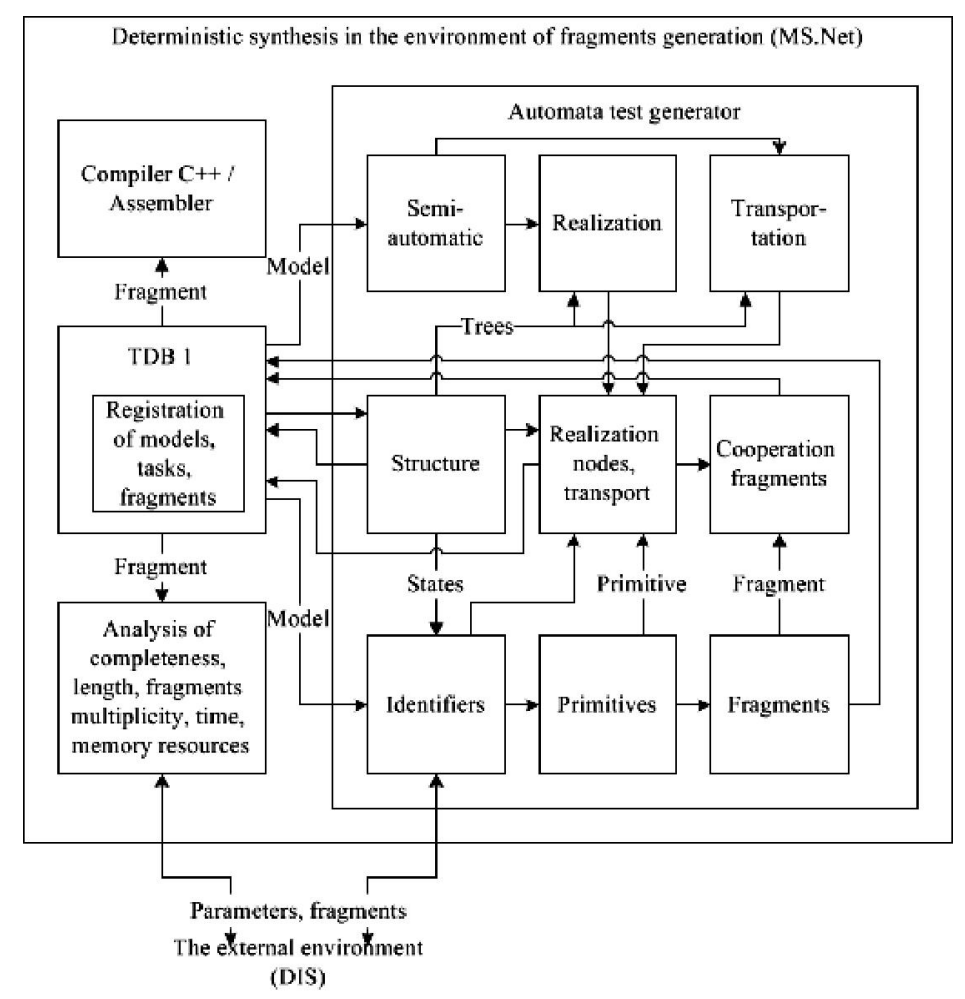

Fig. 1 - Block diagram of a deterministic generator.

Recursive steps of the procedures are performed due to the precedence of the automata models of the DIS structure components, based on the search into the depth/width with the local optimization, accumulating (providing) nodal results.

Preprocessor procedures provide nodal results only once. These results can be reused in order to accelerate the synthesis of behavioral tests.

The basic procedure of deterministic one-fold active synthesis of behavioral tests $T e x_{i}$ and repetitive subsequent testing, performed in test mode for the automata model of the tested DIS component, includes:
1) construction of the test fragments $T f_{\text {RjTiTrk }}$ and synthesis of the complete behavioral test $\mathrm{Tex}_{i}$ modified by the search into the depth/width with local optimization, for example, pseudo-Euler crawl, on the base of narrowed identifiers $T i_{\text {RjTiT }}$, link $L p_{R i T i T r k}$ and test $T p_{\text {RTTiTrk }}$ primitives;

2) recursive building of the inverse compliances $T f_{R j T i T r k}$ and complete behavioral test $T \operatorname{Tex}_{R j T i T r k}$ into the relevant structures of input actions input test semi-automata $T^{-1}\left(a_{i}\right)\left(T f_{i Y}\right)$ and $T^{-1}\left(a_{i}\right)\left(T e x_{i}\right)$ for implementing nodal subnet of automata model $a_{i}$ of tested component; 
3) recursive building of the direct mapping of test fragments $T f_{R j T i T r k}$ and complete behavioral test $T e x_{R T \text { TiTrk }}$ into the relevant structures of output reactions - output test semi-automata $T\left(a_{i}\right)\left(T f_{i Y}\right)$ and $T\left(a_{i}\right)\left(T e x_{i}\right)$ for transporting nodal subnet $T\left(a_{i}\right)$ of automata model $a_{i}$ of the tested component.

The basic procedure of deterministic repeated passive synthesis of behavioral tests $T e x_{i}$ and testing, executed in the operation mode for the automata model $a_{i}$ of the tested DIS component includes:

1) real time input-buffering in a set of analyzed input/output words $W^{\text {"^ }}$ based on the current operation functioning of a component;

2) deterministic treelike search-recognition of

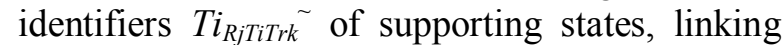
$L p_{\text {RjTiTrk }} \tilde{}$ and test $T p_{\text {RjTiTrk }} \tilde{\text { primitives, }}$ implemented and transported in the automata network on a set of input/output words $W$ "'^ based on identifiers $T i_{R j T i T r k}$, primitives $L p_{R j T i T r k}$ and $T p_{R j T i T k}$, which were previously built by the pre-preprocessor procedure;

3) formation of pseudo-test fragments $T f_{\text {RjTiTrk }}$ (candidates) and structure - automata $a_{i}^{\sim}$ - of the input/output behavior, based on the identification of the supporting states and the existing determinism of automata functions $a_{i}$;

4) deterministic treelike search-recognition of linking $L p_{\text {RiTiTrk }}$ and test $T p_{\text {RiTiTrk }}$ primitives, test fragments $T f_{R j T i T r k}$ based on the formed structure automata $a_{i}^{\sim}-$ of input/output behavior $W^{\prime \prime}$;

5) registration of recognized test fragments $T f_{R j T i T r k}$ and evaluation of private and general completeness $\varphi v_{i}$, length $\lambda \varepsilon_{i}$, multiplicity $\theta v_{i}$ and $\theta v \varepsilon_{i}$, feasibility $\rho \varepsilon_{i}$ and portability $\tau \rho_{i}$ of testing.

The preprocessor procedure of deterministic implementation of the behavioral tests $T e x_{i}$ and testing, performed in the operation and test modes for automata model $a_{i}$ of the tested DIS component in case of forward recursive construction of implemented nodal semi-automata $a R_{T}^{X-1}(a i)$, includes:

1) deterministic alternative, based on the search into the depth/width of the current following unexplored automata model $a_{i}^{\sim}$ with the lowest number in the input nodal reverse subnet $T^{-1}\left(a_{i}\right)$ for automata model $a_{i}$ of the tested component;

2) input/buffering of output nodal implemented semi-automata $a R_{T}^{Y-1}(a \tilde{i})$ of the current reverse nodal subnet $T^{1}\left(a_{i}^{\sim}\right)$ for the current automata model $a_{i}^{\sim}$;

3) alphabetic mapping of the output nodal implemented semi-automata $a R_{T}^{Y-1}(a \tilde{i})$ of the current nodal reverse subnet $T^{-1}\left(a_{i}^{\sim}\right)$ into the input nodal implemented semi-automata $a R_{i}^{X \sim}$ of the current automata model $a_{i}^{\sim}$, representing a set of input words, available in the current input nodal reverse subnet $T^{-1}\left(a_{i}^{\sim}\right)$;
4) composition $a R_{i}^{X \sim o} a_{i}^{\sim}$ of the current input nodal implemented semi-automata $a R_{i}^{X \sim}$ and current automata model $a_{i}$, which generates the modified implemented current automata model $\tilde{a_{i} R j}$;

5) output narrowing $\Downarrow_{Y}\left(a_{i}^{\sim}{ }_{i j}\right)$ of the modified implemented current automata model $a_{i R R}^{\sim}$, resulting into the output nodal implemented semi-automata $a_{i}^{\sim Y}{ }_{R j}$ at the output of current automata model $a_{i}^{\sim}$;

6) minimization $\min \left(a_{i}{ }^{\sim}{ }_{R j}\right)$ of current output nodal implemented semi-automata $a_{i}^{\sim Y}{ }_{R j}$;

7) if not all of the automata models in the input nodal reverse subnet are considered, then go to step 1).;

8) alphabetic mapping of the output nodal implemented semi-automata $a R_{T}^{Y-1}(a i)=a_{i}^{\sim Y_{R j}}$ of the reverse nodal subnet $T^{-1}\left(a_{i}\right)$ of the automata model $a_{i}$ of the tested component into its input nodal implemented semi-automata $a_{i}^{X}$, representing a set of input words, available in the input nodal reverse subnet $T^{1}\left(a_{i}\right)$.

The preprocessor procedure of deterministic transportation of behavioral tests Tex $x_{i}$ and the testing, executed in the operation and test modes for automata model $a_{i}$ of the tested DIS component in case of the reverse recursive construction of transported nodal semi-automata $\operatorname{aTr}_{T(a i)}^{Y}$, includes:

1) deterministic alternative, based on the search into the depth/width of the current following unexplored automata model $a_{i}{ }^{\sim}$ with the largest number in the output direct nodal subnet $T\left(a_{i}\right)$ for the automata model $a_{i}$ of the tested component;

2) input/buffering of the input nodal transported semi-automata $\operatorname{aTr}_{T(a i)}^{X}$ of the current nodal direct subnet $T\left(a_{i}^{\sim}\right)$ for the current automata model $a_{i}$;

3) alphabetic mapping of input nodal transported semi-automata $\operatorname{aTr}_{T(a i)}^{X}$ of the current nodal direct subnet $T\left(a_{i}\right)^{\tilde{}}$ into the output nodal transported semi-automata $a \operatorname{Tr}_{i}^{Y \sim}$ of current automata model $a_{i}^{\sim}$, that represents a set of output words, transported in current output nodal direct subnet $T\left(a_{i}^{\sim}\right)$;

4) composition $a_{i}^{\sim}{ }^{O} a \operatorname{Tr}_{i}^{Y \sim}$ of the current automata model $a_{i}^{\sim}$ and output nodal transported semiautomata $\operatorname{aTr}_{i}^{Y \sim}$, which generates a modified transported automata model $\tilde{a}_{i}^{\sim} T_{r}$;

5) input narrowing $\Downarrow_{X}\left(a_{i}^{\sim} T_{r k}\right)$ of modified transported current automata model $\tilde{a_{i}} \operatorname{Trk}$ resulting into the input nodal transported semi-automata $a_{i}^{\sim X}{ }_{r r k}$ at the input of current automata model $a_{i}^{\sim}$;

6) minimization $\min \left(a_{i}^{\sim X}{ }_{T r k}\right)$ of current input nodal transported semi-automata $a_{i}^{\sim X}{ }_{T r k}$;

7) if not of the all automata models in the output nodal direct subnet are considered, then go to step 1); 
8) alphabetic mapping of the input nodal semiautomata $a \operatorname{Tr}_{T(a i)}^{X}=a_{i}^{\sim X}{ }_{T r k}$ of the direct nodal subnet $T\left(a_{i}\right)$ of the automata model $a_{i}$ of the tested component in its output nodal semiautomata $a_{i}^{Y} T r k$, representing a set of output words, transported in the output nodal direct subnet $T\left(a_{i}\right)$.

Modeling of the evolution generator is based on the expanded set of component implementations of evolutionary-genetic models and methods of passive and active synthesis of behavioral tests Tex , forming the technological evolution structure of the test fragments $T f_{i}$ (see Fig. 2). The composition and communications of structure are defined by the solved evolutionary tasks and relationships between them.

A set of additional procedures for the test tasks execution by the evolutionary generator has a number of features.

The general procedure of preprocessor test synthesis of behavioral tests $\operatorname{Tex}_{i}$ for automata model $a_{i}$ of DIS component is complemented by:

1) the evolutionary definition of identifiers $T i_{i}$ of supporting states, linking $L p_{i}$ and test $T p_{i}$ primitives on their basis;

2) the evolutionary definition of transportable (recognizable) sub-automata $a \operatorname{Tr}_{i}^{Y}$.

The general procedure of preprocessor test synthesis of behavioral tests for the DIS automata network model is supplemented by:

1) evolutionary direct and reverse construction of correspondingly implementing $T^{1}\left(a_{i}\right)$ and transporting $T\left(a_{i}\right)$ nodal subnets;

2) direct evolutionary construction of implementable nodal semi-automata $a R_{T}^{X-1}$ (ai) on the basis of the implementing nodal subnets $T^{-1}\left(a_{i}\right)$;

3) reverse evolutionary construction of transported nodal semi-automata $a \operatorname{Tr}_{T(a i)}^{Y}$ on the basis of the transporting nodal subnets $T\left(a_{i}\right)$.

Evolutionary steps of procedures are executed due to the precedence of the automata models of the DIS structure components, based on the pseudorandom targeted search, with the accumulation (provision) of nodal results.

When the evolutionary generator is used, nodal results are initially provided by the execution of the preprocessor procedures in order to reuse them for the purpose of acceleration of the synthesis of behavioral tests and development.

The basic procedure of evolutionary one-fold active synthesis of behavioral tests $T e x_{i}$ and repetitive subsequent testing, executed in the test mode of the automata model of the tested DIS component, is supplemented by:

1) evolutionary construction of the test fragments $T f_{\text {RjTiTrk }}$ and synthesis of behavioral test $T e x_{i}$ due to the pseudo-random targeted search, based on the restricted identifiers $T i_{R j T i T k}$, linking $L p_{R j T i T r k}$ and test $T p_{R j T i T r k}$ primitives;

2) evolutionary construction of the inverse mapping of test fragments $T f_{R j T i T r k}$ and behavioral test Tex RjTiTrk $_{\text {in }}$ in the revant structures of input actions - input test semi-automata $T^{-1}\left(a_{i}\right)\left(T f_{i Y}\right)$ and $T^{-1}\left(a_{i}\right)\left(T e x_{i}\right)$ in the operating nodal subnet $T^{-1}\left(a_{i}\right)$ of the automata model $a_{i}$ of the tested component;

3) evolutionary building of the direct mapping of the test fragments $T f_{R j T i T r k}$ and behavioral test Tex $x_{R j T i T r k}$ into the relevant structures of output reactions - output test semi-automata $T\left(a_{i}\right)\left(T f_{i Y}\right)$ and $T\left(a_{i}\right)\left(T e x_{i}\right)$ in the transporting nodal subnet $T\left(a_{i}\right)$ of the automata model $a_{i}$ of the tested component.

The basic procedure of the evolutionary repetitive passive synthesis of behavioral tests Tex $_{i}$ and testing, executed in the operation mode of the automata model $a_{i}$ of the tested DIS component is supplemented by:

1) evolutionary search-recognition of identifiers $T i_{R j T i T r k} \tilde{\text { of supporting states, linking } L p_{R j T i T r k} \sim \text { and }}$ test $T p_{R j T i T r k} \sim$ primitives, implemented and transported in the automata network, on a set of

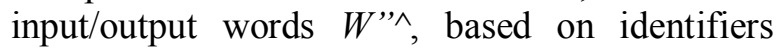
$T i_{\text {RjTiTrk, }}$, primitives $L p_{\text {RjTiTrk }}$ and $T p_{\text {RjTiTrk }}$, previously built according to the preprocessor procedure;

2) evolutionary search-recognition of linking $L p_{\text {RjTiTrk }}$ and test $T p_{R j T i T r k}$ primitives, test fragments $T f_{R j T i T k k}$, based on the formed structure

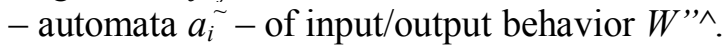

The preprocessor procedure of evolutionary implementation of behavioral tests $T e x_{i}$ and testing, executed in the operation and test modes for automata model $a_{i}$ of the tested DIS component in case of direct evolutionary construction of implemented nodal semi-automata $a R_{T}^{X-1}(a i)$, is complemented by pseudo-random targeted search of the current following unexplored automata model $a_{i}^{\sim}$ with the best evolutionary criteria in the input nodal reverse subnet $T^{l}\left(a_{i}\right)$ of automata model $a_{i}$ of the tested component.

The preprocessor procedure of evolutionary transportation of behavioral tests $\mathrm{Tex}_{i}$ and testing, executed in the operation and test modes for automata model $a_{i}$ of the tested DIS component in case of the reverse evolutionary construction of transported nodal semi-automata $\operatorname{aTr}_{T(a i)}^{Y}$, is supplemented by a pseudo-random targeted search of the current following unexplored automata model $a_{i}^{\sim}$ with the best evolutionary criteria in the output nodal direct subnet $T\left(a_{i}\right)$ of automata model $a_{i}$ of the tested component. 


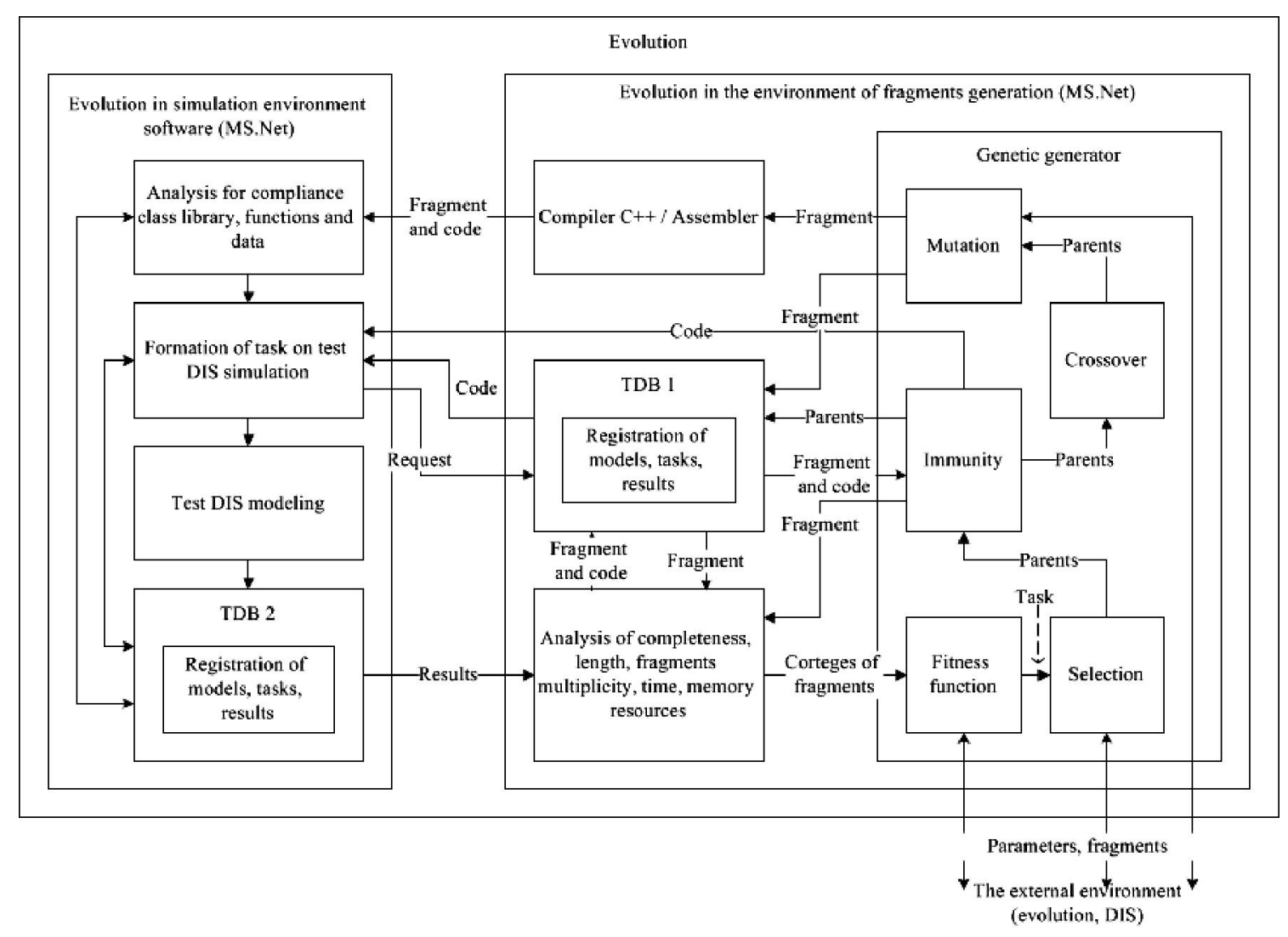

Fig. 2 - Block diagram of an evolutionary generator.

A priori and a posteriori value assessment of the criteria of completeness $\varphi v_{i}$, length $\lambda \varepsilon_{i}$, multiplicity $\theta v_{i}, \theta v \varepsilon_{i}$, feasibility $\rho \varepsilon_{i}$ and portability $\tau \rho_{i}$, defining the synthesis of behavioral tests $\operatorname{Tex}_{i}$, as the conditional control and/or recognition experiment, is applied for the source data and for the received intermediate and final results of deterministic and evolutionary generators.

The agent supervisor ensures the consistent placement and diagnostic functioning of the agent in the environment of the selected DIS component, consistent with its operation functioning.

The basic supervisor procedure of the functioning of an agent $a g_{i}$ is characterized by the following actions:

1) Direct (on behalf of the agent $a g_{i}$ ) or indirect (on behalf of MAS) identification of the target DIS component for agent $a g_{i}$.

2) Remote, direct (on behalf of the agent $a g_{i}$ ) or indirect (on behalf of the MAS) authentication of an agent $a g_{i}$ in the DIS component.

3) Remote, direct or indirect preliminary coordination of required computing resources of the agent $a_{g i}$, in particular, of reactive $a_{g R i}$ and deliberative $a_{g D i}$ components.

4) The internal (agent $a g_{i}$ ) or external (MAS) control of the agent $a g_{i}$ transportation to the selected DIS component through the network DIS environment, in particular, the network of automata NA.
5) If a set of test tasks $\operatorname{Task}_{i}$ of the agent $a g_{i}$ is not empty, then the formation of the next test task task $_{i} \in$ Task $_{i}$ with the initialization of the iterative cycle of the agent ag can take place, otherwise there is the transition to $n .13$.

6) Initialization of iteration of the agent $a g_{i}$, as the formation of the agent model of placement $m_{i}=\left(a_{i}, T i_{i}, T p_{i}, T f_{i}, a R_{i}^{X} a \operatorname{arr}_{i}^{Y}, T^{-1}\left(a_{i}\right), T\left(a_{i}\right)\right.$, $a R_{T}^{X-1}(a i), a \operatorname{Tr}_{T(a i)}^{Y}$ ) (on the initial step - initial model of placement $m_{0 i}$ ) and its test criteria of completeness $\varphi v_{i}$, length $\lambda \varepsilon_{i}$, multiplicity $\theta v_{i}$ and $\theta v \varepsilon_{i}$, feasibility $\rho \varepsilon_{i}$ and portability $\tau \rho_{i}$.

7) If the test task task $k_{i}$ of iterative cycle is solved and the final values for the test criteria of completeness $\varphi v_{i}$, length $\lambda \varepsilon_{i}$, multiplicity $\theta v_{i}$ and $\theta v \varepsilon_{i}$, feasibility $\rho \varepsilon_{i}$ and portability $\tau \rho_{i}$ are received, then there is the transition to $n$. 5 .

8) Formation of the environment $e n_{i}$ of the agentworld in the DIS component, as a part of its tested automata $a_{i}$ and a set of basic places $P l_{i}$, the agent's $a g_{i}$ own model, and also the connection Conn $n_{i}$ between the agent $a g_{i}$ and the environment $e n_{i}$, defining a set of the boundary computing resources $\operatorname{Res}_{i}$ of a component for the corresponding basic places $P l_{i}$.

9) The current authorization of the agent $a g_{i}$ in the DIS component.

10) Specification of the final $q_{i}^{F}$ and formation of the current $q_{i}$ goals of the agent $a g_{i}$, according to the analysis of the status of a model $m_{i}$ 
concerning the part $T i_{i}, T p_{i}, T f_{i}, a R_{i}^{X}, a T^{Y}{ }_{i}$, $T^{-1}\left(a_{i}\right), T\left(a_{i}\right), a R_{T}^{X-1}(a i), a \operatorname{Tr}_{T(a i)}^{Y}$, and also due to the current and final test criteria of completeness $\varphi v_{i}$, length $\lambda \varepsilon_{i}$, multiplicity $\theta v_{i}$ and $\theta v \varepsilon_{i}$, feasibility $\rho \varepsilon_{i}$ and portability $\tau \rho_{i}$. $\rho \varepsilon_{i}$

11) Formation and execution of the strategy $s t_{i}$ of the current goal $q_{i}$ achievement as a choice of a system of the interconnected deterministic and/or evolutionary steps (in the simplest case one step) for creation of the following model $m_{i}{ }^{\prime}=\left(a_{i}, \quad T i_{i}{ }^{\prime}, \quad T p_{i}{ }^{\prime}, \quad T f_{i}{ }^{\prime}, \quad a R_{i}^{X}, \quad \operatorname{aTr}_{i}^{Y}, T^{1}\left(a_{i}\right)^{\prime}\right.$, $\left.T\left(a_{i}\right), \quad a R_{T}^{X}{ }^{\prime}(a i), \quad a \operatorname{Tr}_{T(a i)}^{Y}{ }^{\prime}\right)$ according to the current goal $q_{i}$.

12) The transition to $n .7$.

13) Completion of the current session of the agent $a g_{i}$ with the expectation of the event of nonempty set of the test tasks Task.

Individual program components, their properties, methods, and interfaces can be applied separately. Being included into the parallel diagnostic components, they can be used at the request of this agent and other related MAS agents. Cooperation of the components is specifically determined by the agents, initiating the testing, taking into account the criteria of the results assessment - completeness of the error checking, length, multiplicity, the tests feasibility and portability, computing costs, depending on the allocation environment.

At the system, program and information level of modeling, the last task is performed due to the use of distributed input buffers (cache) of the agents tasks, dynamic (the current state) priorities of the tasks,

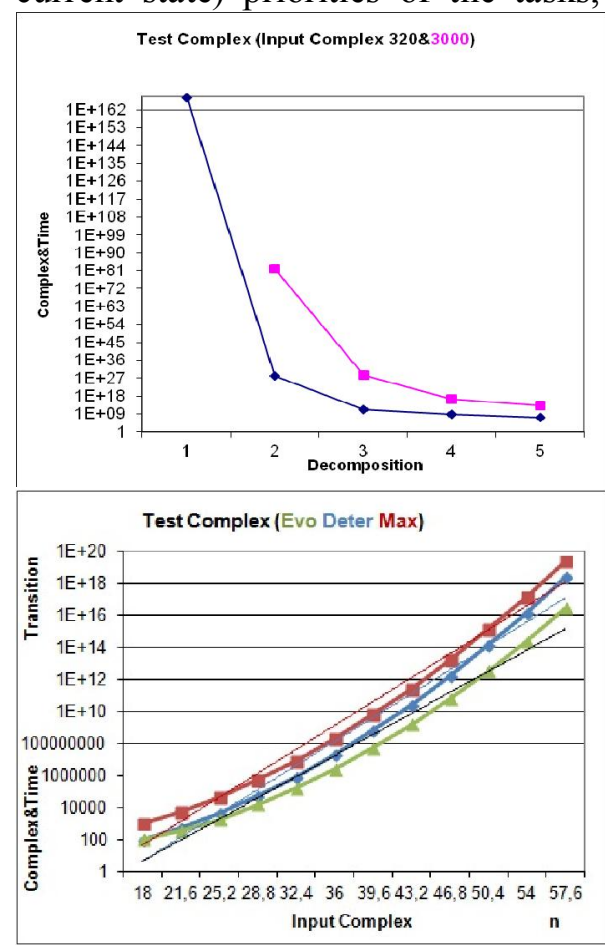

mechanisms of the critical sections of resources, quantization of access to them, transactions in the tasks of behavioral testing and output buffers (cache) of the agents solutions.

At the transportation level, adjacent to the application test level below, interaction of the cooperation agents in the DIS environment can be provided by its own component means of communication, the virtualized for MAS agents as the external transportation interface components.

Functions of the libraries of the MS Visual.Net program modeling environment and special agent libraries, in particular, are used for implementation of the basic programs of generators and supervisor.

Program modeling results allowed to verify the models and evaluate the area of their applicability, in particular, the possibility of transportation of the $N P$-complexity of the synthesis of behavioral tests into the accessible area (see. Fig. 3).

The time of synthesis was reduced, but the length and the tests completeness remained the same, with the use of multi-agent decomposition and evolutionary methods. The time of the test synthesis has been reduced to $92 \%$ (from 95 minutes (100\%) to 8 minutes $(8 \%)$ ) for the experimental DIS mechanisms at the complexity level IPv.4, IPv.6 and IPSec, applying correspondingly the deterministic and evolutionary methods.

Multi-agent synthesis of behavioral tests and testing based on verification of the functional mapping of automata models of the DIS components increases the multiversion of the problem solution and allows to take into account the DIS features.

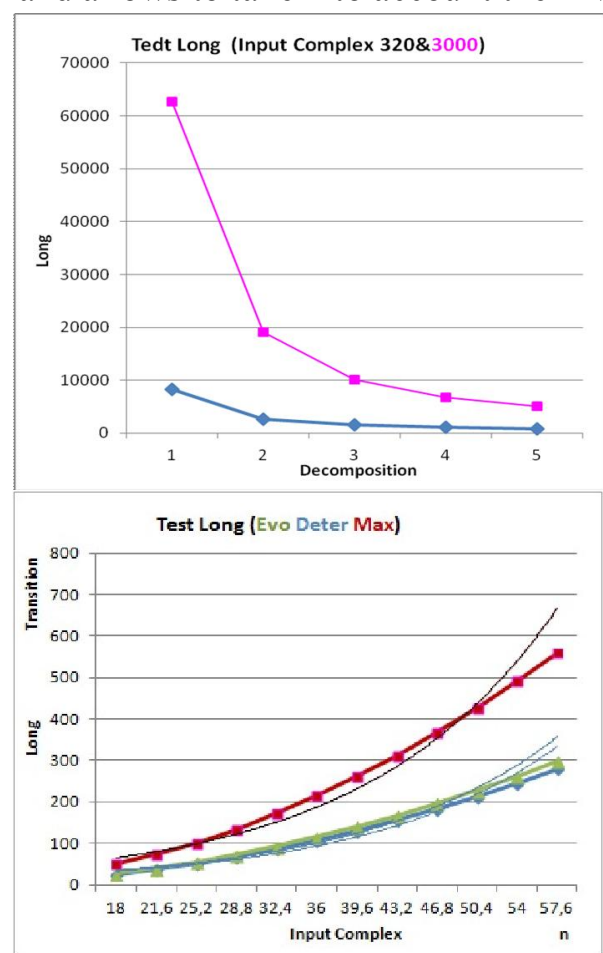

Fig. 3 - The dependence of the complexity and length of the tests on the coefficient of expansion and the complexity of the input components. 


\section{CONCLUSION}

The proposed agent model of the behavioral testing allows to execute the distributed synthesis of behavioral tests on the basis of deterministic and evolutionary models and methods, and also to implement them during the operation and test DIS control taking into account the test conditions of the DIS components.

Combined methods due to the decomposition of testing processes in the distributed MAS allow to reduce the time of synthesis of tests to $90 \%$, increase the adequacy of representation of the structure and interactions of real DIS in case of allowable computing costs of the cluster level of laboratory network, used in the background.

The exponential complexity, restricting the use of behavioral tests, and dimensionality are polynomially reduced due to the component (DIS) and agent (MAS) decomposition.

The implementation of the MAS conceptual model, executed at the structural and functional and program-algorithmic level in the MS Visual.Net environment, showed the possibility and feasibility of further research and practical work in this direction.

\section{REFERENCES}

[1] A.S. Tanenbaum, M.V. Steen, Distributed Systems: Principles and Paradigms, third ed., Prentice Hall Press, 2013, 705 p.

[2] G. Coulouris, J. Dollimore, T. Kindberg, G. Blair, Distributed Systems: Concepts and Design, 5th ed., Boston: Addison-Wesley, 2011, 1067 p.

[3] I. Turchenko, V. Kochan, A. Sachenko, Neuralbased recognition of multi-parameter sensor signal described by mathematical model, International Journal of Computing, (3) 2 (2004), pp. 140-147.

[4] V.I. Hahanov et al., Design and Verification of Digital Systems on Chips. Virology \& System Virology, Studies Benefits, Kharkov Nat. Univ. Radioelektronics, Kharkov, Ukraine, A New Word, 2010, 527 p. (in Russian).

[5] H. Tahbildar and B. Kalita, Automated software test data generation: direction of research, International Journal of Computer Science \& Engineering Survey (IJCSES), (2) 1 (2011), pp. 99-120.

[6] V. Hrusha, O. Ossolinsky, A. Sachenko, R. Kochan, Distributed on-line temperature measurement and control system, International Journal of Computing, (6) 2 (2007), pp. 62-67.

[7] S.J. Russell, P. Norvig, Artificial Intelligence: a Modern Approach, Prentice-Hall, Inc. A Simon \& Schuster Company Englewood Cliffs, New Jersey, 2010, $1095 \mathrm{p}$.
[8] Y. Shoham, K. Leyton-Brown, Multiagent Systems. Algorithmic, Game-Theoretic, and Logical Foundations, 2010, 532 p.

[9] G. Rojek, R. Cięciwa, K. Cetnarowicz, Algorithm of behavior evaluation in multi-agent system, in Proceedings of the International Conference on Computational Science ICCS'2005: 5-th Int. Conference: Atlanta, GA, USA, (May 22-25, 2005) Pt. 3-ed., in: Vaidy S. Sunderam [et al.], Lecture Notes in Computer Science LNCS, vol. 3516. Berlin; Heidelberg: Springer-Verlag, 2005, pp. 711-718.

[10] R.M. Hierons, K. Bogdanov, J.P. Bowen, R. Cleaveland, J. Derrick, J. Dick, M. Gheorghe, M. Harman, K. Kapoor, P. Krause, G. Luttgen, A.J.H. Simons, S. Vilkomir, M.R. Woodward, H. Zeda, Using formal specifications to support testing, $A C M$ Comput. Surv., (41) (2009), pp. 9:1-9:76.

[11] M.J. Rutherford, Adequate System-Level Testing of Distributed Systems, A thesis submitted to the Faculty of the Graduate School of the University of Colorado, 2006, $158 \mathrm{p}$.

[12] K. Sinha, Structural Complexity and its Implications for Design of Cyber-Physical Systems, Massachusetts Institute of Technology, Engineering Systems Division, January 3, 2014, 342 p.

[13] N.K. Jha and S. Gupta, Testing of Digital Systems, Cambridge University Press, 2003, $1018 \mathrm{p}$.

[14] W.K. Lam, Hardware Design Verification: Simulation and Formal Method-based Approaches, Prentice Hall, 2005, 624 p.

[15] V.B. Kudryavtsev, I.S. Grunskii, V.A. Kozlovskii, Analysis and synthesis of abstract automata, Journal of Mathematical Sciences, (169) 4 (2010), pp. 481-532.

[16] I. Grunsky, O. Kurganskyy, I. Potapov, Languages representable by Vertex-labeled graphs, in Proceedings of the 30-th International Symposium on Mathematical Foundations of Computer Science, (2005), vol. 3618, pp. 435-446.

[17] A.A. Shchurov, R. Mařík, A formal approach to distributed system tests design, International Journal of Computer and Information Technology, (3) 4 (2014), pp. 696-705.

[18] R.M. Hierons, H. Ural, The effect of the distributed test architecture on the power of testing, The Computer Journal, (51) 4 (2008), pp. 498-510.

[19] O. Baldellon, J.-C. Fabre, M. Roy, Minotor: monitoring timing and behavioral properties for dependable distributed systems, in Proceedings of the 19-th IEEE Pacific Rim International Symposium on Dependable Computing 
PRDC'2013, Vancouver, Canada, (December 2013), $10 \mathrm{p}$.

[20] G. Jervan, R. Ubar, Z. Peng, P. Eles, Test Generation: A Hierarchical Approach, Chapter in System-level Test and Validation of Hardware/Software Systems, Springer Series in Advanced Microelectronics, (17) (2005), pp. 67-81.

[21] A.M. Khamis, M.R. Girgis, A.S. Ghiduk, Automatic software test data generation for spanning sets coverage using genetic algorithms, Computing and Informatics, (26) (2007), pp. 383-401.

[22] J. Hudec, E. Gramatová, An efficient functional test generation method for processors using genetic algorithms, Journal of Electrical Engineering, (66) 4 (2015), pp. 185-193.

[23] P. Bernardi, E. Sanchez, M. Schillaci, G. Squillero, M. Sonza Reorda, An evolutionary methodology to enhance processor software-based diagnosis, in Proceedings of the IEEE Congress on Evolutionary Computation, Vancouver BC, (July 16-21, 2006), pp. 859864.

[24] M. Harman, P. McMinn, a theoretical \& empirical analysis of evolutionary testing and hill climbing for structural test data generation, in Proceedings of the International Symposium on Software Testing and Analysis, London, (912 July 2007), pp. 73-83.

[25] S.K. Singh, S. Sabharwal and J.P. Gupta, A novel approach for deriving test scenarios and test cases from events, Journal of Information Processing Systems, (8) 2 (2012), pp. 213-240.

[26] Z. Houhamdi, B. Athamena, Structured integration test suite generation process for multi-agent system, Journal of Computer Science, (7) 5 (2011), pp. 690-697.

[27] C. Nguyen, A. Perini, P. Tonella, Goal-oriented testing for MASs, Int. J. Agent-Oriented Software Eng., (4) (2010), pp. 79-109.

[28] S. Yu, J. Ai, Software test data generation based on multi-agent, International Journal of Software Engineering and its Applications, (4) 1 (2010), pp. 67-74.

[29] A.S. Sugak, A.N. Martynyuk, Evolutionary network model of testing for distributed information systems, Elecrotehnical and Computer Systems, Odessa, Ukraine, 16(92) (2014), pp. 71-77. (in Russian).

[30] A.S. Sugak, A.N. Martynyuk, Multi-agent systems of behavioral diagnosis for distributed information system, Elecrotehnical and Computer Systems, Odessa, Ukraine, 19(95) (2015), pp. 187-194. (in Russian).

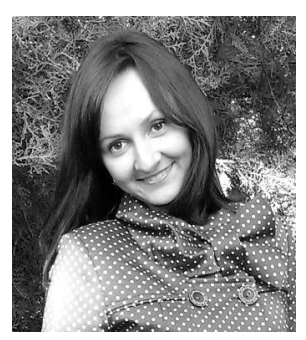

Anna Sugak, graduated Odessa National Polytechnic University, Information systems Department. Now she works as assistant of the Department of Computerized Control Systems at Odessa National Polytechnic University.

Research interests: monitoring and diagnostics of computing devices, synthesis test for discrete devices.

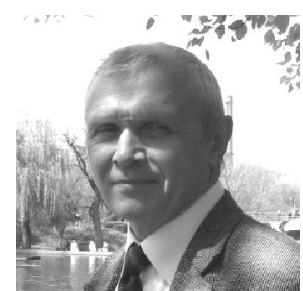

Ph.D., Oleksandr Martynyuk, graduated Odessa Polytechnic Institute, Electronic Computing Devices Department. Now he works as associate professor of Department of Computer's Intellectual Systems and Networks at Odessa National Polytechnic University.

Research interests: protocol analysis and verification of computer networks, test synthesis for computer systems.

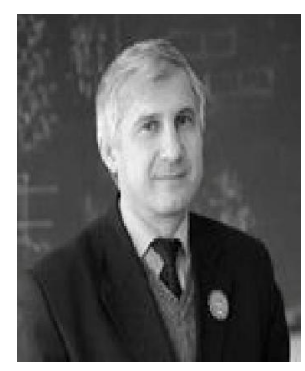

Professor Oleksandr Drozd, graduated Odessa Polytechnic Institute, Electronic Computing Devices Department. Now he works as Professor of Department of Computer's Intellectual Systems and Networks at Odessa National Polytechnic University.

Research interests: functional (working) diagnosis of computing devices, accuracy of approximate calculations, dependability of critical system and application. 